\title{
FREQUENCY ASSESSMENT OF EMERGENCE OF EXTENSIVELY DRUG RESISTANT SALMONELLA TYPHI STRAINS IN QUETTA, BALOCHISTAN
}

\author{
Shafia Nasir, Naveed Asif, Oroosa Nasir*, Sunila Tashfeen Arif, Muhammad Azam \\ Combined Military Hospital Quetta/National University of Medical Sciences (NUMS) Pakistan, *Combined Military Hospital Malir/National \\ University of Medical Sciences (NUMS) Pakistan
}

\begin{abstract}
Objective: To determine frequency of multidrug resistant and extensive drug resistant strains of Salmonella typhi and Salmonella paratyphi in Quetta, Balochistan of Pakistan

Study Design: Cross-sectional study.

Place and Duration of Study: Department of Microbiology, Combined Military Hospital Quetta, Pakistan, from Mar 2019 to Mar 2020.

Methodology: A total of two thousand seven hundred and sixty $(n=2760)$ suspected cases of typhoid fever, irrespective of age and gender reporting at the hospital during one year were enrolled. Isolates were cultured and identified using standard microbiological procedures. Antimicrobial sensitivity against the typhoidal Salmonellae was determined using Kirby-Bauer disc diffusion method. All the extensively drug-resistant isolates were confirmed by Vitek 2 system.

Results: A total of 173 (6.3\%) cultures showed positive results. Mean age of culture positive cases was $18.9 \pm 11.1$ years. Out of 173 positive blood cultures, 136 (78.6\%) were of males and 37 (21.1\%) were from females. Antibiotic susceptibility results showed that $166(96 \%)$ of isolates were Salmonella typhi. Multi-drug resistant strains were observed in $104(60.1 \%)$ and extensive drug resistant strains were observed in 81 (46.8\%) isolates.

Conclusion: Frequencies of MDR (60.1\%) and XDR (46.8\%) strains of S. typhi isolates from confirmed cases of enteric fever were remarkable in Quetta, Balochistan. We recommend empiric therapy with azithromycin in patients with uncomplicated disease and therapy with carbapenam for complicated cases of enteric fever acquired in Karachi or Balochistan.
\end{abstract}

Keywords: Enteric fever, Extensive drug resistance, Multidrug resistance.

This is an Open Access article distributed under the terms of the Creative Commons Attribution License (http://creativecommons.org/licenses/by/4.0), which permits unrestricted use, distribution, and reproduction in any medium, provided the original work is properly cited.

\section{INTRODUCTION}

A serotype of Salmonella enterica known as Salmonella typhi (S. typhi) is typically responsible for enteric fever. Other Salmonella serotypes, particularly S. enterica serotypes and paratyphi A, B, or $C$ can cause similar syndrome ${ }^{1}$. Several factors including the infecting species and infectious dose influence pathogenesis of enteric fever ${ }^{2}$. Evidence suggests that $S$. typhi are human-restricted pathogens that have been evolved in response to environmental pressures, especially virulent clones that may exist. However further studies are needed to understand the specific virulence properties of this pathogen ${ }^{3}$. Potential role of typhoid toxin in disease pathogenesis is an area

Correspondence: Dr Shafia Nasir, Department of Microbiology, Combined Military Hospital, Quetta Pakistan

Received: 14 Aug 2020; revised received: 22 Aug 2020; accepted: 26 Aug 2020 of investigative focus. Typhoid toxin is a pyramidal holotoxin that contains a homopentamer of pertussis-like toxin B subunit (PltB) at its base, with pertussis-like toxin A (PltA; an adenosine diphosphateribosyl transferase) in the center, and cytolethal distending toxin (CdtB; a DNase that results in cell cycle arrest) at the apex. $S$. typhi have uniquely evolved this toxin to adapt to humans and exclusively express the toxin while in vacuoles 4 . Data on number of $S$. typhi organisms required to cause disease have been obtained from human volunteer studies and from epidemiologic investigations. In general, the attack rate is higher with greater infectious dose and the shorter incubation period gets 5 . Multidrug resistant (MDR) strains of $S$. typhi (resistant to three first line drugs i.e., Ampicilin, chloramphenicol and trimethoprim-sulfamethoxazole) have already been observed worldwide ${ }^{6}$. Typhoid fever 
showing extensively drug-resistance (XDR) is increasing in Pakistan. An outbreak of XDR S. typhi was recognized in November 2016 in Pakistan and is still considered as ongoing7. It increases the dreads of failure of antibiotic treatment all over the world 8 . One study reported that during 2016 \& 2018, 29 cases of typhoid fever have been reported to travel to or from Pakistan. About $17.2 \%$ cases had extensively drug-resistance typhoid 9 . Through another study, it was observed that $73.6 \%$ resistant Salmonella strains to different antibiotics were found. Overall resistance for both S. typhi and S. paratyphi A was observed $64 \%$ from ampicillin, 63\% from chloramphenicol 39\% from ciprofloxacin and $28 \%$ from third generation ceftriaxone and cefixime ${ }^{10}$.

Present study was planned to determine frequency of MDR and XDR strains of $S$. typhi and S. paratyphi in Quetta, Balochistan, since high number of people are consistently moving between Karachi and Balochistan. This research would help us in gathering evidence about the resistance pattern of $S$. typhi in reference to XDR in Quetta. This shall be helpful in guiding and recommending empiric treatment. Gathered data would also serve as an important feedback to public health authorities to act accordingly to reduce further spread of ongoing outbreak.

\section{METHODOLOGY}

This descriptive cross-sectional study and data was collected over period of one year from March 2019 to March 2020 at CMH Quetta. Study design was approved from local Institutional Ethics Committee (CMH-QTA-IRB/043 Dated 11 August 2020). All the patients, irrespective of age and gender, form both the indoor and outdoor departments, who were suspected of typhoid fever and planned for blood culture for confirmation of diagnosis were enrolled into the study through non-probability consecutive sampling. All the cases with confirmed diagnosis of typhoid fever on blood culture were further analyzed for antimicrobial susceptibility testing. All those cases where $S$. typhi or $S$. paratyphi was not isolated as the causative organism were excluded from further antimicrobial susceptibility testing. All those cases were also excluded who were not willing to participate in the study. A well-informed written consent was obtained from every participant. Target sample size (minimum of $\mathrm{n}=$ 152) was determined taking $\alpha=0.05$, an acceptable margin of error $\mathrm{d}$ as 0.06 . Proportion of XDR was estimated as $17.2 \%$, based on a previous study ${ }^{10}$. Suspected cases of typhoid fever were those who had history of prolonged fever (temperature $>101^{\circ} \mathrm{F}$ for $>1$ week) along with other symptoms like headache, nausea, loss of appetite, diarrhea and vomiting. Blood samples were withdrawn using $10 \mathrm{cc}$ disposable syringe under strict aseptic measures. All positive blood cultures were inoculated on routine culture media, i.e., Blood and Mac Conkey's Agar and were incubated for 24 hours at 35-37 degree centigrade. Growth of organisms was identified through appropriate technique of colonial morphology, gram staining and biochemical analysis and with API 105 and API 20E. Antibiotic susceptibility pattern was determined for enlisted antibiotics by KirbyBauer disk diffusion method on Mueller-Hinton agar with standard antimicrobial disks. Study outcome was assessed in terms of susceptibility pattern as sensitive and resistant as per CLSI (Clinical and Laboratory Standard Institute) Guidelines, 2019*. Multidrug resistant (MDR) strain was labeled if $S$. typhi were resistant to three first line drugs (Ampicillin, chloramphenicol and trimethoprim-sulfamethoxazole). Extensively drug resistant strain (XDR) was labeled if $S$. typhi were resistant to three first line drugs (Ampicillin, chloramphenicol and trimethoprimsulfamethoxazole) along with 3rd generation cephalosporins and flouroquinolones. Data was analyzed using SPPS version 22. Numeric data were presented as mean \pm SD and ranked data as frequency and percentages. Results obtained were stratified across gender and different age groups. Chi-square test was applied as the test of significance and a $p \leq 0.05$ was set as significant.

\section{RESULTS}

During study period two thousand seven hundred and sixty $(n=2760)$ suspected cases of 
typhoid fever reported at our hospital. Blood culture was carreid out in all the suspected cases. There were total of $173(6.3 \%)$ cases in whom culture showed positive results. All the culture positive isolates were further analyzed. Mean age of the culture positive cases was $18.9 \pm 11.1$ years. Out of 173 blood cultures, 136 (78.6\%) were of males and $37(21.1 \%)$ were from females. Most of the culture poistive cases $(87.9 \%)$ were below age of 30 years (table-I). Antibiotic susceptibility results showed that $166(96 \%)$ of isolates were $S$. typhi. MDR strains were observed in 104 (60.1\%)

Table-I: Demographic data of study cases.

\begin{tabular}{|c|c|}
\hline Age (Mean years \pm SD) & $18.9 \pm 11.1$ \\
\hline \multicolumn{2}{|l|}{ Age Groups, n (\%) } \\
\hline$\leq 18$ years & $73(42.2 \%)$ \\
\hline $19-30$ years & $79(45.7 \%)$ \\
\hline $31-40$ years & $15(8.6 \%)$ \\
\hline$>40$ years & $6(3.5 \%)$ \\
\hline \multicolumn{2}{|l|}{ Gender, n (\%) } \\
\hline Males & $136(78.6 \%)$ \\
\hline Females & $37(21.4 \%)$ \\
\hline \multirow{2}{*}{\multicolumn{2}{|c|}{$\begin{array}{l}\text { Table-II: Culture and aI } \\
\text { results. } \\
\text { Pathogen Type, n (\%) }\end{array}$}} \\
\hline & \\
\hline \multirow{3}{*}{\multicolumn{2}{|c|}{$\begin{array}{l}\text { Salmonella typhi } \\
\text { Salmonella typhi }\end{array}$}} \\
\hline & \\
\hline & \\
\hline \multirow{2}{*}{$\begin{array}{l}\text { Positive } \\
\text { Negative }\end{array}$} & $104(60.1 \%)$ \\
\hline & $69(39.9 \%)$ \\
\hline \multicolumn{2}{|c|}{ Extensive Drug Resistant, n (\%) } \\
\hline Positive & $81(46.8 \%)$ \\
\hline Negative & $92(53.2 \%)$ \\
\hline
\end{tabular}

of isolates and XDR strains were observed in 81 $(46.8 \%)$ isolates (table-II). A uniform distribution of MDR strains was observe $\mathrm{d}$ across gender and different age groups $(p=0.506$ and 0.544 respectively, table-III). XDR strains were observed in higher proportions in cases with $\leq 18$ and $>40$ years of age when compared to those with 19-40 years of age, however, the difference was not statistically significant $(p=0.320$ and 0.055 respectively, table-IV).

\section{DISCUSSION}

Likelihood of typhoid fever should be considered in patients who are febrile and living in, visting to or travelling from endemic areas. In all the suspected cases of enteric fever blood and stool culture are considered mandatory investigations ${ }^{11}$. Presnet study results showed that out of total two thousand seven hundred and sixty $(n=2760)$ suspected cases of typhoid fever, blood culture was poistive for $S$. typhi and S. paratyphi in $173(6.3 \%)$ cases. Evidence suggests that culture of most specimens is not highly sensitive and when cultures are negative or not available, as in some settings with limited resources, diagnosis of enteric fever is often made presumptively on the

Table-III: Distribution of multidrug resistant strains $(\%)$ among gender and different age groups.

\begin{tabular}{|c|c|c|c|c|}
\hline \multirow{2}{*}{\multicolumn{2}{|c|}{ Variables }} & \multicolumn{2}{|c|}{ Multidrug Resistant } & \multirow{2}{*}{$\begin{array}{c}p- \\
\text { value }\end{array}$} \\
\hline & & Present & Absent & \\
\hline \multirow{4}{*}{ 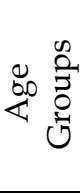 } & $<18$ years & $48(46.2 \%)$ & $25(36.2 \%)$ & \multirow{4}{*}{0.544} \\
\hline & $19-30$ years & $43(41.3 \%)$ & $36(52.2 \%)$ & \\
\hline & $31-40$ years & $9(8.7 \%)$ & $6(8.7 \%)$ & \\
\hline & $>40$ years & $4(3.8 \%)$ & $2(2.9 \%)$ & \\
\hline \multirow{2}{*}{$\frac{\overline{0}}{0}$} & Males & $80(76.9 \%)$ & $56(81.2 \%)$ & \multirow{2}{*}{0.506} \\
\hline & Females & $24(23.1 \%)$ & $13(18.8 \%)$ & \\
\hline
\end{tabular}

Table-IV: Distribution of extensive drug resistant strains (\%) among gender and different age groups.

\begin{tabular}{|c|c|c|c|c|}
\hline \multirow{2}{*}{\multicolumn{2}{|c|}{ Variables }} & \multicolumn{2}{|c|}{$\begin{array}{c}\text { Extensive Drug } \\
\text { Resistant }\end{array}$} & \multirow{2}{*}{$\begin{array}{c}p- \\
\text { value }\end{array}$} \\
\hline & & Present & Absent & \\
\hline \multirow{4}{*}{ 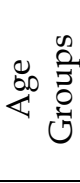 } & $<18$ years & $40(49.4 \%)$ & $33(35.9 \%)$ & \multirow{4}{*}{0.055} \\
\hline & $19-30$ years & $34(42 \%)$ & $45(48.9 \%)$ & \\
\hline & $31-40$ years & $3(3.7 \%)$ & $12(13 \%)$ & \\
\hline & $>40$ years & $4(4.9 \%)$ & $2(2.2 \%)$ & \\
\hline \multirow{2}{*}{$\begin{array}{l}\overline{0} \\
\stackrel{\tilde{U}}{0} \\
\stackrel{0}{0}\end{array}$} & Males & $61(75.3 \%)$ & 75 (81.5\%) & \multirow{2}{*}{0.320} \\
\hline & Females & $20(24.7 \%)$ & $17(18.5 \%)$ & \\
\hline
\end{tabular}

basis of a protracted febrile illness without other explanation. Empiric therapy is often appropriate in the absence of an alternative diagnosis because of the risk for severe sequelae with untreated enteric fever; nevertheless, it is imperative to recognize that the clinical syndrome of enteric fever is nonspecific, and positive predictive value of a clinical diagnosis even in high-burden settings is typically $<50 \% 12$. Bone marrow culture is considered as the most sensitive (>90\%) diag- 
nostic modality but is rarely indicated in routine clinical practice ${ }^{13}$.

In the present study, antibiotic susceptibility result showed that 166 (96\%) of isolates were $S$. typhi. MDR strains were observed in 104 (60.1\%) of cases with a uniform distribution of MDR strains observed across gender and different age groups ( $p=0.506$ and 0.544 respectively. Numerous outbreaks of MDR strains have been reported in the endemic regions including Africa, China, and Southeast Asia ${ }^{14}$. Prevalence rates vary widely across the regions and ranging from $10-80 \% 15,16$. Analysis of isolates from all different geographical regions and their genome sequencing has identified a predominant MDR, S. Typhi strain, H58, that has spread throughout Africa and Asia, and driving ongoing MDR epidemics ${ }^{17}$. As of 2018, approximately 75\% of strains from Africa remain MDR, without significant change over the past 15 years ${ }^{18}$. In a subsequent Surveillance of Enteric Fever in Asia Project study, a minority of strains from India, Nepal, and Bangladesh were MDR, while the majority of strains from Pakistan continued to show multidrug resistance ${ }^{19}$.

Present study result showed XDR strains were observed in 81 (46.8\%) of cases. XDR strains observed in higher proportions in cases with $\leq 18$ and $>40$ years of age when compared to those with 19-40 years of age, however, the difference was not statistically significant $(p>0.055)$. Extensively-drug resistance has been detected in several regions of South Asia including Pakistan. It was concomitant to several epidemics in late 1980s \& early $1990 \mathrm{~s}^{20}$. According to updated data of World Health Organization, in Pakistan, few health officials say that in Nov. 2016 to Dec. 2018 epidemic of extensively drug-resistance typhoid fever, affected $>5200$ people ${ }^{21}$. Outbreak started in Pakistan in 201618,22. By the end of 2018, over 5000 cases of this XDR, S. typhi strain were reported, with imported cases in United Kingdom and United States 23,24 . Strain remains susceptible to azithromycin and carbapenems, which are the main treatment options for this strain.
Ideally, definitive antimicrobial therapy for enteric fever is based on results of susceptibility testing. When treating presumptively for enteric fever or before results of susceptibility testing are available, appropriate options for empiric therapy depend on the severity of disease and the risk of infection with an antibiotic-resistant isolate. In patients with severe or complicated disease acquired in Pakistan (those living in the endemic regions or those following recent travel to Pakistan), we suggest empiric therapy with a carbapenem (e.g., meropenem). This is because of an ongoing outbreak of XDR S. typhi in this region ${ }^{25}$. In patients with uncomplicated or mild disease acquired in Pakistan (those living in the endemic regions or those following recent travel to Pakistan), we suggest empiric therapy with azithromycin, which achieves excellent intracellular concentrations and has established efficacy. Resistance to azithromycin remains rare and it is expected to have activity against XDR isolates acquired in Pakistan.

In summary, a remarkable proportion of $S$. typhi isolates from confirmed cases of enteric fever demonstrated MDR and XDR strains in Quetta, Balochistan. This is an alarming situation as frequent domestic travelling would increase the risk of spread of these XDR strains in other parts of the country. Several countries in Europe and in USA already started screening of suspected cases travelling from Pakistan. In the light of present study results, we recommend empiric treatment with azithromycin for patients with uncomplicated or mild disease acquired in Karachi or in Balochistan and for patients with severe or complicated enteric fever acquired in these regions; we suggest empiric therapy with a carbapenem. Study results also serve as an important feedback to public health authorities to act proactively to reduce further spread of ongoing outbreak. We also suggest conducting large scale population-based studies across Pakistan in order to get better information about antibiotic susceptibility pattern of $S$. typhi and paratyphi in different regions of Pakistan. 


\section{ACKNOWLEDGMENT}

We wish to acknowledge all the studied patients for their cooperation. We also wish to pay special gratitude to our families and friends for their continuous support and motivation.

\section{CONCLUSION}

A significant proportion of $S$. typhi isolates from confirmed cases of enteric fever demonstrated MDR (60.1\%) and XDR (46.8\%) strains in Quetta, Balochistan. We recommend empiric therapy with azithromycin in patients with uncomplicated disease and therapy with carbapenam for complicated cases of enteric fever acquired in Karachi or Balochistan.

We also suggest conducting large scale population-based studies across Pakistan in order to get better information about antibiotic susceptibility pattern of $S$. typhi and paratyphi in different regions of Pakistan.

\section{CONFLICT OF INTEREST}

This study has no conflict of interest to be declared by any author.

\section{REFERENCES}

1. Trawinski $H$, Wendt $S$, Lippmann N, Heinitz S, von Braun A, Lübbert $C$, et al. Typhoid and paratyphoid fever. Z Gastroenterol 2020; 58(2): 160-70.

2. Verma S, Senger S, Cherayil BJ, Faherty CS. Spheres of influence: insights into salmonella pathogenesis from intestinal organoids. Microorganisms 2020; 8(4): 504-09.

3. Hong YP, Wang YW, Huang IH, Liao YC, Kuo HC, Liu YY, et al. Genetic relationships among multidrug-resistant Salmonella enterica serovar Typhimurium strains from humans and animals. Antimicrob Agents Chemother 2018; 62(5): e00213-18.

4. Fowler CC, Chang SJ, Gao X. Emerging insights into the biology of typhoid toxin. Curr Opin Microbiol 2017; 35(2): 70-73.

5. Darton TC, Zhou L, Blohmke CJ. Blood culture-PCR to optimise typhoid fever diagnosis after controlled human infection identifies frequent asymptomatic cases and evidence of primary bacteraemia. J Infect 2017; 74(4): 358-66.

6. Mashe T, Gudza-Mugabe M, Tarupiwa A. Laboratory characterisation of Salmonella enterica serotype Typhi isolates from Zimbabwe, 2009-2017. BMC Infect Dis 2019; 19(1): 487-92.

7. Klemm EJ, Shakoor S, Page AJ. Emergence of an extensively drug-resistant salmonella enterica serovar typhi clone harboring a promiscuous plasmid encoding resistance to fluoroquinolones and third-generation cephalosporins. mBio 2018; 9(1): e00105-18.

8. Yates JA, Rao SR, Walker AT, Esposito DH, Sotir M, LaRocque RC. Characteristics and preparation of the last-minute traveler: analysis of vaccine usage in the Global Trav Epi Net Consortium. J Travel Med 2019; 26(6): 1-6.
9. Chatham-Stephens K, Medalla F, Hughes M, Appiah GD, Aubert RD, Caidi H. Emergence of extensively drug-resistant salmonella typhi infections among travelers to or from Pakistan - United States, 2016-2018. MMWR Morb Mortal Wkly Rep 2019; 68(1): 11-13.

10. Iqbal M, Mirza S, Hassan MA. Prevalence and current trends of antimicrobial resistance among Salmonella typhi and Salmonella paratyphi A in children. Isra Med J 2019; 11(1): 41-45.

11. Shane AL, Mody RK, Crump JA. 2017 Infectious Diseases Society of America Clinical Practice Guidelines for the Diagnosis and Management of Infectious Diarrhea. Clin Infect Dis 2017; 65(12): e45-e80.

12. Mogasale V, Ramani E. What proportion of Salmonella Typhi cases are detected by blood culture? A systematic literature reviews. Ann Clin Microbiol Antimicrob 2016; 15(1): 32-37.

13. Gilman RH, Terminel M, Levine MM. Relative efficacy of blood, urine, rectal swab, bone-marrow, and rose-spot cultures for recovery of Salmonella typhi in typhoid fever. Lancet 1975; 1(7918): 1211-13.

14. Yan M, Li X, Liao Q, Li F, Zhang J, Kan B, et al. The emergence and outbreak of multidrug-resistant typhoid fever in China. Emerg Microbes Infect 2016; 5(6): e62.

15. Wain J, Hendriksen RS, Mikoleit ML, Keddy KH, Ochiai RL. Typhoid fever. Lancet 2015; 385(9973): 1136-45.

16. Marks F, von Kalckreuth V, Aaby P, Sarkodie Y, Tayeb MA, Ali $\mathrm{M}$, et al. Incidence of invasive salmonella disease in sub-Saharan Africa: a multicentre population-based surveillance study. Lancet Glob Health 2017; 5(3): e310-23.

17. Wong VK, Baker S, Pickard DJ, Parkhil J, Page AJ, Feasey NA. Phylogeographical analysis of the dominant multidrug-resistant H58 clade of Salmonella Typhi identifies inter- and intracontinental transmission events. Nat Genet 2015; 47(6): 632-39.

18. Britto CD, Wong VK, Dougan G. A systematic review of antimicrobial resistance in Salmonella enterica serovar Typhi, the etiological agent of typhoid. PLoS Negl Trop Dis 2018; 12(10): e0006779.

19. Barkume C, Date K, Saha SK, Qamar FN, Sun D, Andrews JR, et al. Phase I of the Surveillance for Enteric Fever in Asia Project (SEAP): An Overview and Lessons Learned. J Infect Dis 2018; 218(4): S188-94.

20. Parry CM, Ribeiro I, Walia K, Rupali P, Baker S, Basnyat B, et al. Multidrug resistant enteric fever in South Asia: unmet medical needs and opportunities. Bio Med J 2019; 364(1): k5322

21. Khan E. Drug Resistant Typhoid Fever: An emerging Public Health Crisis. Rawal Med J 2019; 44(1): 1-3.

22. Qamar FN, Yousafzai MT, Khalid M, Kazi AM, Lohana H, Karim S, et al. Outbreak investigation of ceftriaxone-resistant Salmonella enterica serotype Typhi and its risk factors among the general population in Hyderabad, Pakistan: a matched casecontrol study. Lancet Infect Dis 2018; 18(12): 1368-75.

23. Extensively Drug-Resistant Typhoid Fever in Pakistan https:// wwwnc.cdc.gov/travel/notices/alert/xdr-typhoid-feverpakistan

24. Stephens KC, Medalla F, Hughes M, Appiah GD, Aubert RD, Caidi $\mathrm{H}$, et al. Emergence of extensively drug-resistant salmonella typhi infections among travelers to or from Pakistan United States, 2016-2018. MMWR Morb Mortal Wkly Rep 2019; 68(1): 11-13.

25. Kariuki S, Gordon MA, Feasey N, Parry CM. Antimicrobial resistance and management of invasive Salmonella disease. Vaccine 2015; 33(3): C21-29. 